\section{OPEN ACCESS}

Edited by: Jianxun Ding,

Chinese Academy of Sciences, China

Reviewed by:

Xianghong Luan,

Texas A\&M University, United States

He Liu,

Jilin University, China

Jinlin Song,

Chongqing Medical University, China

*Correspondence:

Chang Liu

changliudentist@gzhmu.edu.cn

${ }^{\dagger}$ These authors have contributed equally to this work and share first authorship

Specialty section: This article was submitted to Biomaterials,

a section of the journal

Frontiers in Materials

Received: 09 November 2020 Accepted: 15 January 2021

Published: 12 March 2021

Citation:

Yi P, Liang J, Huang F, Zhao Z, Zhou X, Gao Q, Huang M, Chen B, Guo Z and Liu C (2021) Composite System of 3D-

Printed Polymer and Acellular Matrix Hydrogel to Repair

Temporomandibular Joint Disc.

Front. Mater. 8:621416.

doi: 10.3389/fmats.2021.621416

\title{
Composite System of 3D-Printed Polymer and Acellular Matrix Hydrogel to Repair Temporomandibular Joint Disc
}

Ping $\mathrm{Yi}^{1+}$, Jiadi Liang ${ }^{2 \dagger}$, Futing Huang ${ }^{1}$, Zuodong Zhao ${ }^{1}$, Xiaohui Zhou ${ }^{1}$, Qianya Gao ${ }^{1}$, Min Huang ${ }^{1}$, Baoyi Chen ${ }^{1}$, Zhenzhao Guo ${ }^{3}$ and Chang Liu ${ }^{1 *}$

${ }^{1}$ Guangzhou Key Laboratory of Basic and Applied Research of Oral Regenerative Medicine, Department of Orthodontics, Affiliated Stomatology Hospital of Guangzhou Medical University, Guangzhou, China, ${ }^{2}$ Center of Stomatology, Shunde Hospital of Southern Medical University, Foshan, China, ${ }^{3}$ Guangzhou Key Laboratory of Basic and Applied Research of Oral Regenerative Medicine, Affiliated Stomatology Hospital of Guangzhou Medical University, Guangzhou, China

Tissue engineering is a promising approach to restore or replace a damaged temporomandibular joint (TMJ) disc. However, constructing a scaffold that can mimic biomechanical and biological properties of the natural TMJ disc remains a challenge. In this study, three-dimensional (3D) printing technology was used to fabricate polycaprolactone (PCL)/polyurethane (PU) scaffolds and PU scaffolds to imitate the region-specific biomechanical properties of the TMJ disc. The scaffolds were coated with polydopamine (PDA) and combined with a decellularized matrix (dECM). Then, rat costal chondrocytes and mouse L929 fibroblasts, respectively, were suspended on the composite scaffolds and the biological functions of the cells were studied. The properties of the scaffolds were characterized by scanning electron microscopy (SEM), X-ray photoelectron spectroscopy (XPS), contact angle analysis, and biomechanical testing. To verify the biocompatibility of the scaffolds, the viability, proliferation, and extracellular matrix (ECM) production of the cells seeded on the scaffolds were assessed by LIVE/DEAD staining, Cell Counting Kit-8 assay, biochemical content analysis, immunofluorescence staining, and qRT-PCR. The functionalized hybrid scaffolds were then implanted into the subcutaneous space of nude mice for 6 weeks, and the regenerated tissue was evaluated by histological staining. The biomechanical properties of PCL/PU and PU scaffolds were comparable to that of the central and peripheral zones, respectively, of a native human TMJ disc. The PDA-coated scaffolds displayed superior biomechanical, structural, and functional properties, creating a favorable microenvironment for cell survival, proliferation, ECM production, and tissue regeneration. In conclusion, 3D-printed polymer scaffolds coated with PDA and combined with dECM hydrogel were found to be a promising substitute for TMJ disc tissue engineering.

Keywords: temporomandibular joint disc, tissue engineering, decellularized extracellular matrix, three-dimensional (3D), polydopamine 


\section{INTRODUCTION}

Temporomandibular disorder (TMD) has been reported to affect $10 \%-15 \%$ of the population, and only $5 \%$ of patients seek treatment (Lim and Sbhalang, 2010; Gonçalves et al., 2011). As a major component of the temporomandibular joint (TMJ), the disc usually undergoes degenerative changes, such as displacement without reduction and perforation in patients with severe TMD, and these pathological changes greatly compromise normal jaw movement and functions, including the ability to eat and speak (Eiji and Theo, 2003). The TMJ disc has poor regeneration capability due to the limited vascularization. At the advanced stage of TMD, the only option for the treatment of the damaged disc is surgical resection (Miloro and Henriksen, 2010; Renapurkar, 2018). Though discectomy can partially relieve symptoms, permanent recovery will not occur due to the development of condylar remodeling (Eriksson and Westesson, 1992; Abdala-Junior et al., 2018). To avoid progressive degenerative changes of the affected TMJ, placement of an interpositional material is highly recommended. However, replacement of the damaged disc with alloplastic or autogenous graft leads to a range of complications, which considerably limit the clinical application (Dolwick, 2007; Dimitroulis, 2011).

Tissue engineering is emerging as a promising approach to attenuate symptoms of TMD, even repair or potentially replace the injured discs. Scaffolding materials play a crucial role in providing mechanical support and biochemical signals for tissue regeneration. Until now, numerous natural materials and synthetic polymers have been used to engineer the disc ( $\mathrm{Wu}$ et al., 2014; Kobayashi et al., 2015; Bousnaki et al., 2018; Wang et al., 2018; Moura et al., 2020). Natural scaffolds, especially the tissue-specific decellularized extracellular matrix (dECM), generally possess high biocompatibility and recognition domains to facilitate cell adhesion, proliferation, and differentiation through inherent biochemical cues within native tissues, while the poor mechanical strength impedes its application in regenerating load-bearing tissues (Brown et al., 2012; Liang et al., 2020). On the other hand, the lack of mechanical properties of these biomaterials can be overcome by combining them with synthetic polymers to form composite structures with superior mechanical strength, biocompatibility, and biodegradability (Pina et al., 2019).

At present, the main methods used to prepare scaffolds are electrospinning, freeze-drying, three-dimensional (3D) printing, and so on (Acri et al., 2019). 3D printing technology is an ideal way to fabricate biomimetic scaffolds with a desired shape, suitable mechanical properties, and a wellcontrolled microstructure to facilitate cell infiltration and distribution (Legemate et al., 2016; Tamay et al., 2019). Various synthetic polymers, including poly(lactic-co-glycolic acid) (PLGA), polylactic acid (PLA), polycaprolactone (PCL), and polyurethane (PU), have been used for printing soft and hard tissues due to their fine-tunable properties (Daly et al., 2017). Recently, PCL and PU have been widely investigated for regeneration of cartilaginous tissue because they possess excellent biocompatibility and adequate mechanical properties, which can be maintained for a long time (Bahcecioglu et al., 2019; Theodoridis et al., 2019; Wen et al., 2019). However, scaffolds made of these materials have smooth surfaces, are hydrophobic, and lack cell recognition sites; thus, their ability to promote cell adhesion, proliferation, and differentiation is poor (Zhu et al., 2002). To address this challenge, various surface modification techniques have been developed to mediate cell adhesion and subsequent cell responses (Richbourg et al., 2019). Recently, functionalizing biomaterials with different biomacromolecules, such as proteins, peptides, or growth factors, has attracted much attention since this approach may evoke cell- and tissuespecific reactions (Lee et al., 2014; Tarafder et al., 2016; Capellato et al., 2020). Compared to other chemical conjugation methods, the polydopamine (PDA) coating tends to be nontoxic, solvent-free, and time-saving (Qiu et al., 2018). This innovative surface modification method has therefore been extensively explored for applications in engineering various hard and soft tissues, such as bone, cartilage, blood vessels, nerves, and muscle (Qian et al., 2018; Bock et al., 2020; GodoyGallardo et al., 2020; Wei et al., 2020).

In the present study, we aimed to develop a biomimetic composite scaffold of 3D printed synthetic polymers and tissue-specific dECM hydrogel to repair the TMJ disc. The composite scaffold developed can provide an ideal physical and biochemical microenvironment to promote cell attachment, proliferation, differentiation, and tissue regeneration by combining PDA-coated PCL/PU and PU scaffolds with tissue-specific decellularized ECM hydrogel derived from porcine TMJ discs. Therefore, this biomimetic composite scaffold holds potential to be a promising approach for TMJ disc tissue engineering.

\section{MATERIALS AND METHODS}

\section{Fabrication of the 3D Printed Scaffolds}

Based on the region-specific mechanical properties of TMJ disc, 3D printed scaffolds were fabricated using a mixture of PCL (Sigma, USA) and PU (Tecoflex, EG-100A, United States) at a ratio of 1:1, according to the results of our pilot study. PCL and PU particles were heated at $100^{\circ} \mathrm{C}$ for $30 \mathrm{~min}$ in the miniature twin screw extruder (MiniJet, HAAKE MiniJet, Germany), then the mixture was extruded at a speed of $100 \mathrm{r} / \mathrm{min}$ at a temperature of $140^{\circ} \mathrm{C}$. PCL/PU and PU scaffolds with a pore size of about $350 \mu \mathrm{m}$ were fabricated using a $3 \mathrm{D}$ printer (Regenovo, China). The materials were melted at $170^{\circ} \mathrm{C}$ in printing chamber, printed through a 22 gauge metal needle at pressure $0.45 \mathrm{Mpa}$ and deposition speed of $0.5 \mathrm{~mm} / \mathrm{s}$. Printed scaffolds were immersed in a dopamine solution $(2 \mathrm{mg} / \mathrm{ml}, \mathrm{pH}$ 8.5 ) at room temperature for $16 \mathrm{~h}$ under continuous stirring (Jo et al., 2013) and then rinsed several times with deionized water. Samples were used after vacuum drying for $24 \mathrm{~h}$. Scaffolds coated with PDA were classified as "modified" groups and were referred to as PDA-PCL/PU and PDA-PU scaffolds, respectively. The scaffolds without PDA coating were classified as "original" groups. 


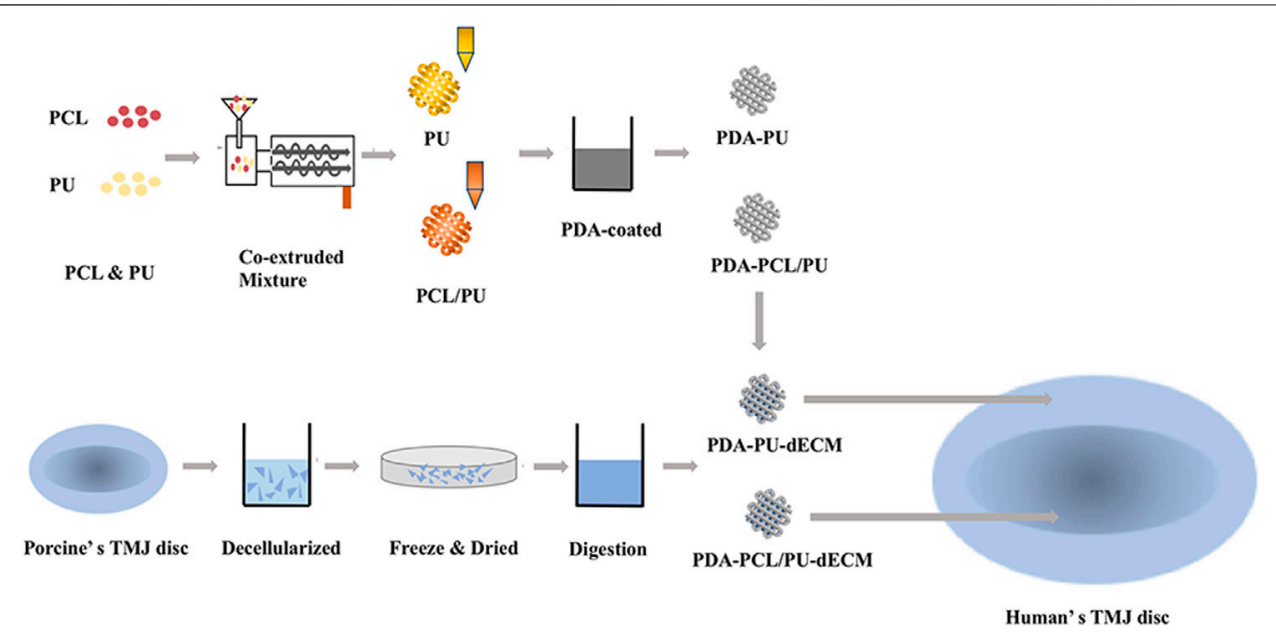

FIGURE 1 | Schematic illustration of the construction process of the composite scaffolds of the experimental design.

\section{Characterization of 3D Printed Scaffolds}

The morphology of the scaffolds was visualized by scanning electron microscopy (SEM, Thermo Fisher Scientific, United States). Samples were sputter-coated with gold at $20 \mathrm{~mA}$ for $60 \mathrm{~s}$, mounted on aluminum stubs, fixed with carbon tape, and observed under a SEM at an accelerating voltage of $10 \mathrm{kV}$. The chemical composition of the scaffolds was analyzed by X-ray photoelectron spectroscopy (XPS, Thermo Fisher K-Aloha, United States). The containment C1s hydrocarbon peak at $284.7 \mathrm{eV}$ was applied to calibrate the binding energies, and the sensitivity factors of $\mathrm{C} 1 \mathrm{~s}$, N1s, and O1s were quantitatively calculated. The water contact angle of the scaffolds was used to evaluate the hydrophobicity of the scaffolds. Briefly, the same amount of water was dropped onto the surface of each scaffold at room temperature, and the image was captured using a contact angle analyzer (JY-PH, JINHE, China).

\section{Preparation of the Composite Scaffolds Preparation of the Hydrogel}

The decellularization process of TMJ disc was carried out as described in prior study (Liang et al., 2020). Briefly, the minced tissue pieces were placed into a hypotonic Tris- $\mathrm{HCl}$ buffer followed by four cycles of freezing and thawing. These samples were treated with $0.5 \%$ trypsin solution for $24 \mathrm{~h}$ and then with nuclease solution $(50 \mathrm{U} / \mathrm{ml}$ DNase and $1 \mathrm{U} / \mathrm{ml}$ RNase, $\mathrm{pH}$ 7.5) at room temperature for $4 \mathrm{~h}$. Afterward, these samples were treated with $1 \%$ Triton X-100 for $48 \mathrm{~h}$ and washed with deionized water for at least 3 days. The dECM powder was digested with $0.1 \%$ pepsin in $0.01 \mathrm{M} \mathrm{HCl}$ at room temperature for $48 \mathrm{~h}$. The flowable solution was neutralized by adding $0.1 \mathrm{M}$ $\mathrm{NaOH}$, followed by adding $10 \times$ PBS to adjust the physiological acidity and salinity. The pregel solution was diluted to the predetermined concentration $(8 \mathrm{mg} / \mathrm{ml})$ using $1 \times$ PBS for subsequent study, since this concentration has been proven to be the most cells-friendly concentration in a preceding study (Liang et al., 2020).

\section{Fabrication of the Composite Scaffolds}

The dECM solution $(8 \mathrm{mg} / \mathrm{ml})$ was infused into the $3 \mathrm{D}$ printed scaffolds using a dropper and then incubated in a $37^{\circ} \mathrm{C}$ incubator for $30 \mathrm{~min}$ to complete the gelation of the hydrogel. The size of the scaffolds used in the subsequent cell experiments corresponded to the size of the well plates, e.g., when a cell experiment was performed with 48 -well plates, the scaffold size was matched to that of the 48-well plate size. A schematic illustration of the preparation procedure of scaffolds is presented in Figure 1.

\section{Mechanical Tests of the Composite Scaffolds}

Mechanical tests were performed as described previously (Legemate et al., 2016), using Instron mechanical testing instrument (Instron 3,342, Instron, Norwood, MA, United States). Cylinders with $5 \mathrm{~mm}$ diameter and $3 \mathrm{~mm}$ height were prepared for compression test. Bone-shaped scaffolds with $1 \mathrm{~mm}$ thickness and $25 \mathrm{~mm}$ length were printed for tensile test. All scaffolds tested were combined with dECM hydrogel. Compression and tensile mechanical tests were performed at $0.25 \mathrm{~mm} / \mathrm{min}$ after samples had been preconditioned with 15 cycles with strain ranging from 0 to $10 \%$ strain.

\section{Cell Culture Experiments Cells Culture}

L929 cells (i-CELL, China) were cultured in Dulbecco's Modified Eagle's Medium (DMEM) (Hyclone, China) supplemented with $10 \% \mathrm{FBS}$ and $1 \%$ penicillin-streptomycin in an atmosphere containing $5 \% \mathrm{CO}_{2}$ at $37^{\circ} \mathrm{C}$. Primary rat chondrocytes isolated from 4-week-old specific pathogen free rat (SPF), according to an established method (Barbero et al., 2004). Briefly, costal cartilage fragments were digested with $0.2 \%$ collagenase at $37^{\circ} \mathrm{C}$ overnight. Cells were collected from the digested solution and cultured using DMEM/F-12 (Hyclone, China) supplemented with 10\% FBS and $1 \%$ penicillin-streptomycin in a humidified incubator with $5 \%$ 
TABLE 1 | Primer of qPCR.

\begin{tabular}{ll} 
Target gene & \multicolumn{1}{c}{ Primer sequences } \\
\hline Col I (mouse) & Forward: 5'-ATGCCGCGACCTCAAGATG-3' \\
& Reverse: 5'-TGAGGCACAGACGGCTGATA-3' \\
GAPDH (mouse) & Forward: 5'-TGTGTCCGTCGTGGATCTC-3' \\
& Reverse: 5'-TTGCTGTTGAAGCGCAGGA-3' \\
Col II (rat) & Forward: 5'-AATTGGRGRGGACATAGGG-3' \\
& Reverse: 5'-AAGTATTGGGTCCTIGGG-3' \\
Sox 9 (rat) & Forward: 5'-AGGAAGCTGGCAGACCAGTA-3' \\
& Reverse: 5'-ACGAAGGGTCTCTCTCGCT-3' \\
GAPDH (rat) & Forward: 5'-TATGACTCTACCCACGGCAAGT-3' \\
& Reverse: 5'-ATACTCAGCACCAGCATCACC-3'
\end{tabular}

$\mathrm{CO}_{2}$ at $37^{\circ} \mathrm{C}$. Cells at passage 2 were used for all experiments. All animal experiments were approved by Guangzhou Medical University (No. GY2020-044) and conducted according to standard guidelines for the use and care of laboratory animals.

\section{Cell Seeding onto Scaffolds and Viability Assay}

The sterilized composite scaffolds were placed in 48-well plates. L929 and chondrocytes, respectively, were implanted at a density of $1.0 \times 10^{4}$ cells/scaffold to construct cell-laden composite scaffolds and cultured with the growth medium suitable for growth of each type of cells. The viability of the cells on the composite scaffolds was evaluated using a LIVE/DEAD staining kit (BestBio, China) at 1 and 7 days of culture following the manufacturer protocol. The stained cells were observed by confocal laser scanning microscope (Leica TCS SP8, Germany). Cell proliferation was quantified by the Cell Counting Kit-8 assay (CCK8, Dojindo, Japan) at 1, 4, and 7 days of culture and measured at $450 \mathrm{~nm}$ wavelength in a microplate reader (Thermo Scientific, Shanghai, China).

\section{Differentiation and Maturation of Cells Gene Expression Analysis}

Cell-loaded composite scaffolds were cultured in 6-well plates for 14 days, then total RNA was extracted using a TaKaRa MiniBEST Universal RNA Extraction Kit (TaKaRa Biotechnology, Japan). The content and purity of RNA were measured using NanoDrop 2000 (Thermo Fisher, United States). Total RNA (1 $\mu \mathrm{g})$ was reverse-transcribed into cDNA using a PrimeScript TM RT reagent Kit (TaKaRa Biotechnology, Japan). Reversetranscribed cDNA was subjected to quantitative real-time PCR (SYBR Premix Ex Taq, TaKaRa, Japan) using specific primers targeting Sox 9, collagen type II (ColII), collagen type I (ColI), and GAPDH according to the manufacturer's instructions. GAPDH was used as a housekeeping gene. The primer sequences of the target genes are shown in Table $\mathbf{1}$.

\section{Immunofluorescence Staining and Analysis}

Cell-loaded composite scaffolds were cultured in 24-well plates for 14 days, then immunofluorescence staining was used to visualize the deposition of Col I and Col II, respectively. Briefly, the samples were gently washed 3 times with PBS, fixed in 4\% paraformaldehyde (PFA) for $15 \mathrm{~min}$, and immersed in $0.1 \%$ Triton X-100 for $20 \mathrm{~min}$. Then the samples were blocked with $5 \%$ bovine serum albumin
(BSA) for $30 \mathrm{~min}$ and incubated overnight in a primary antibody solution diluted with $1 \%$ BSA in PBS with rabbit anti-Col I and rabbit anti-Col II (1:100, Abcam, USA) at $4^{\circ} \mathrm{C}$. After washing 3 times with PBS, the scaffolds were incubated in the dark with the secondary antibody (1:200, Goat anti-rabbit $\operatorname{IgG}(\mathrm{H}+\mathrm{L})$, Affinity, China) for $30 \mathrm{~min}$. Finally, samples were stained with 4',6-diamidino-2-phenylindole (Dapi, Beyotime, China) for $15 \mathrm{~min}$ in the dark. The images were taken using a confocal laser scanning microscope (Leica TCS SP8, Germany).

\section{Biochemical Content Analysis}

Cell-laden composite scaffolds were cultured in 24-well plates for 14 days, and the biochemical content of sulfated glycosaminoglycans (GAG) and collagen was assessed as described in a previous study (Romanazzo et al., 2018). Briefly, the scaffolds were digested in $1 \mathrm{ml}$ of papain mixture $(125 \mu \mathrm{g} / \mathrm{ml}$ papain in $0.1 \mathrm{M}$ sodium acetate, $5 \mathrm{mML}$-cysteine HCL, and $0.05 \mathrm{M}$ EDTA) at $65^{\circ} \mathrm{C}$ for $18 \mathrm{~h}$ and then were centrifuged at $10,000 \mathrm{rpm}$ for $10 \mathrm{~min}$ to collect supernatant for quantification tests. Using a chondroitin sulphate standard, the dimethylmethylene blue dye binding assay was utilized to quantify sulfated GAG. Total collagen was determined by measuring the hydroxyproline content. Samples were hydrolyzed at $110^{\circ} \mathrm{C}$ for $18 \mathrm{~h}$ in $38 \% \mathrm{HCl}$ diluted in $\mathrm{H}_{2} \mathrm{O}$ and then examined using a chloramine- $\mathrm{T}$ assay with a hydroxyproline:collagen ratio of 1:7.69.

\section{Scaffolds Implantation and In Vivo Analysis}

Cell-loaded composite scaffolds were implanted into subcutaneous pockets in the dorsal surface of 4 -week-old female nude mice to compare the ability of chondrogenesis and fibrogenesis between modified groups and original groups. Briefly, cylindrical composite scaffolds with $5 \mathrm{~mm}$ in diameter and $1 \mathrm{~mm}$ in height were prepared as described above, and $2 \times 10^{6}$ cells were seeded on each scaffold to construct cell-laden composite scaffold. At 6 weeks after implantation, the mice were sacrificed and all implants were retrieved. Prior to sectioning, the implants were embedded in optimal cutting temperature (OCT) compound (Tissue-Tek ${ }^{\circledR}$, Sakura, Japan) and then sectioned transversely at a thickness of $10 \mu \mathrm{m}$. Tissue sections were stained with Alcian blue (AB) and Picrosirius red (PR) to evaluate chondrogenesis and fibrogenesis of the scaffolds, respectively.

\section{Statistical Analysis}

All values were reported as mean \pm standard deviation (mean \pm SD). At least three samples per group were selected for statistical analysis. Statistical analysis was performed with GraphPad Prism 7 (GraphPad Software, Inc., La Jolla, CA). Statistical significance was analyzed using Student's $t$-test. The significant difference for all statistical analyses was defined as $p<0.05$.

\section{RESULTS}

\section{Characterization of the Scaffolds}

A color change to dark brown in the modified groups indicated the successful surface modification with PDA coating. Microscopically, SEM analysis showed that surface morphology of the original scaffolds was remarkably altered 
A
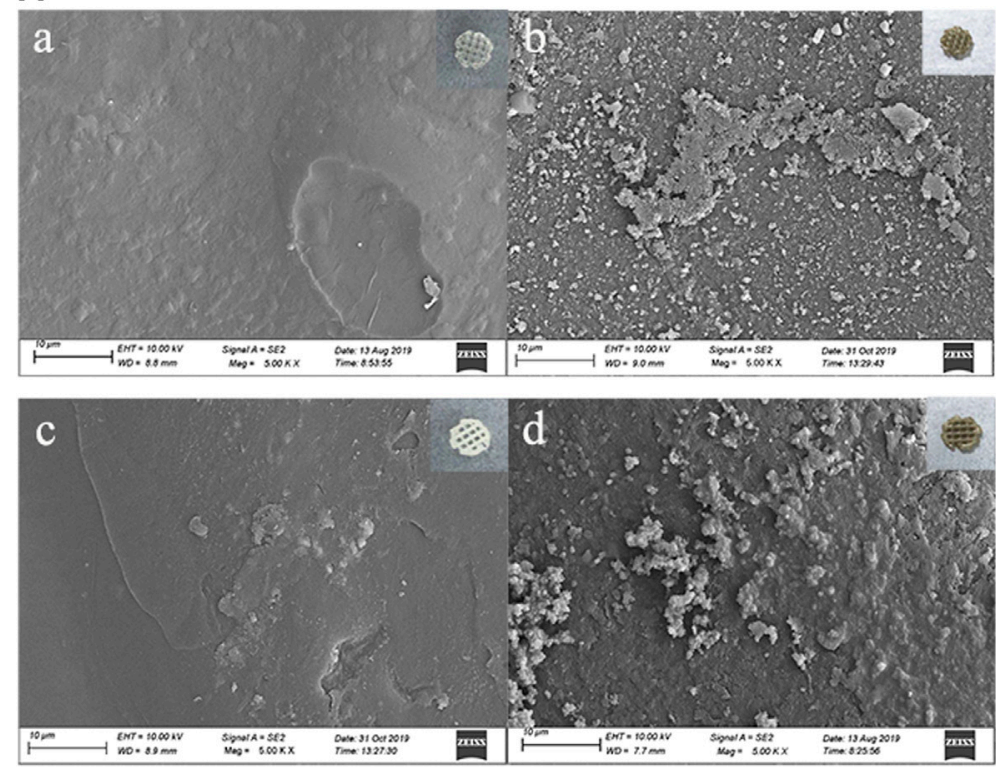

B

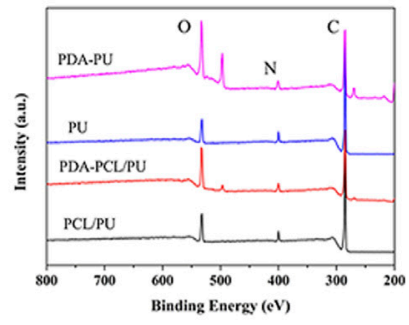

C

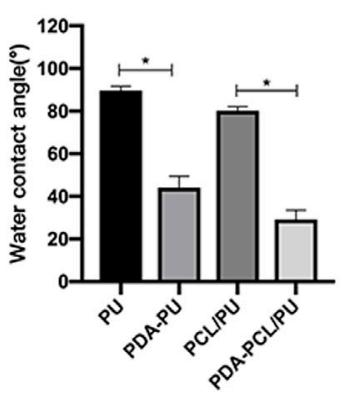

FIGURE 2 | Characterization of the 3D printed scaffolds. (A) SEM micrographs and gross images of the scaffolds: (A) PU; (B) PDA-PU; (C) PCL/PU; (D) PDA-PCL/ PU. The gross images at the upper right corner. (B) XPS spectra of scaffolds. (C) Water contact angle of scaffolds ( $n=3$, $\left.{ }^{\star} p<0.05\right)$.

TABLE 2 | Surface chemical composition of scaffolds.

\begin{tabular}{lcccc}
\hline Sample & $\mathbf{C}(\%)$ & $\mathbf{O}(\%)$ & $\mathbf{N}(\%)$ & $\mathbf{N} / \mathbf{C}$ \\
\hline PU & 83.26 & 11.37 & 5.36 & 0.06 \\
PDA-PU & 70.20 & 23.91 & 5.89 & 0.08 \\
PCL/PU & 80.63 & 14.40 & 4.97 & 0.06 \\
PDA-PCL/PU & 70.00 & 23.35 & 6.65 & 0.10 \\
\hline
\end{tabular}

after PDA coating (Figure 2A). Compared with the smooth surfaces of original groups, spherical aggregates were observed on the surfaces in the modified groups.

The surface chemical compositions of the scaffolds were analyzed by XPS. The spectra showed the existence of carbon $(\mathrm{C} 1 \mathrm{~s}, 284.5 \mathrm{eV})$, oxygen $(\mathrm{O} 1 \mathrm{~s}, 531.0 \mathrm{eV})$, and nitrogen $(\mathrm{N} 1 \mathrm{~s}$, $399.6 \mathrm{eV}$ ) atoms in all scaffolds (Figure 2B). The nitrogen/ carbon $(\mathrm{N} / \mathrm{C})$ ratio in the modified groups was higher than that in the original groups, mainly because PDA contains a large number of nitrogen atoms (Table 2).

The water contact angle was measured to evaluate the efficiency of PDA attachment on original scaffolds. The surfaces of PCL/PU and PU scaffolds became relatively hydrophilic as shown with the significant decrease in water contact angle from $80.20^{\circ}$ to $29.13^{\circ}$ and $89.67^{\circ}$ to $44.70^{\circ}$, after PDA coating (Figure 2C).

The tensile modulus and compressive modulus of the modified groups were significantly higher than those of the original groups $(p<0.05)$. The compression modulus of PDA-PCL/PU (15.970 Mpa) and PDA-PU (6.617 Mpa) scaffolds was similar to that of the middle region (about $15 \mathrm{Mpa}$ ) and the surrounding area (about $5 \mathrm{Mpa}$ ) of human TMJ disc, respectively (Figure 3).

\section{Biocompatibility of the Scaffolds}

The results of cell viability examined by LIVE/DEAD staining are shown in Figure 4A. The living cells, colored green, were the dominant population of seeded cells and primarily resided in the hydrogel at day 1 and day 7. As determined by the amount of green fluorescence, the cell density in the modified groups remarkably increased after 7 days of culture. The proliferation of costal chondrocytes and L929 cells in the scaffolds was evaluated by the CCK-8 assay (Figures 4B,C). The optical density (OD) values increased from day 1 to 7 in all groups, suggesting the composite scaffolds were suitable for cell growth and nutrient transmission. Significantly higher OD values can be found in modified groups compared to original groups at 4 and 7 days $(p<0.05)$, indicating that cell proliferation was enhanced by the PDA coating.

\section{Cell Differentiation and Maturation}

The expression level of chondrocyte and fibroblast marker genes was evaluated with qPCR after 14 days of culture. The modified groups exhibited significant upregulation of the chondrogenicspecific markers (Sox 9 and Col II) and fibrous-specific marker (Col I) compared with the original groups $(p<0.05)$ (Figure 5). These results indicated that the cell differentiation and maturation were promoted in the modified groups.

\section{Immunofluorescence Staining and Biochemical Study}

The presence of collagens in the cell-laden composite scaffolds after 14 days of culture was visualized by immunofluorescence staining. (Figure 6: Col-I [green] and Col-II [red]). The number 
A

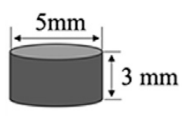

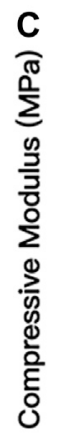

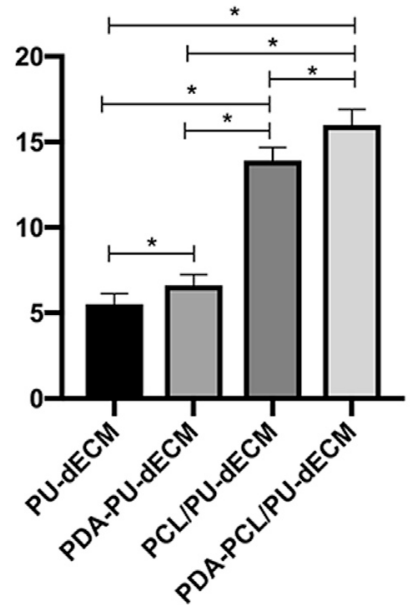

B

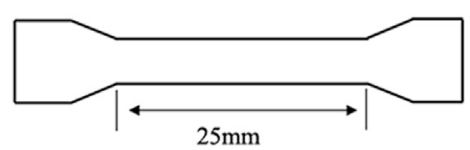

D

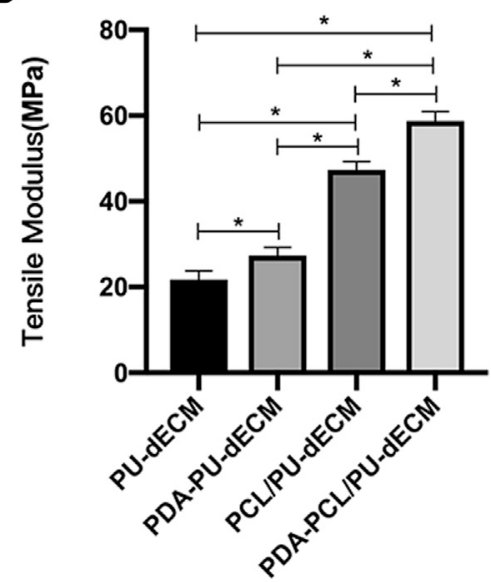

FIGURE 3 |Compressive and tensile modulus of the scaffolds. (A) Graphical representation of the compressive test specimen. (B) Graphical representation of the tensile test specimen. (C) Compressive modulus of the scaffolds. (D) Tensile modulus of the scaffolds. ( ${ }^{\star}$ Comparison between groups, $n=5,{ }^{*} p<0.05$ ).

of cells positive for Col I and Col II in the modified groups was remarkably higher than that in the original groups, suggesting larger amounts of collagens were synthesized in the modified groups. Quantification of collagen and GAG content was carried out using biochemical analysis and the result was shown in Figure 7. There were significantly greater amounts of collagen and GAG deposition in the modified groups than the original groups $(p<0.05)$. These results indicated that the surface modification with PDA was beneficial to the secretion of these two major components of the extracellular matrix.

\section{In Vivo Cartilage and Fibrous Tissue Regeneration}

Sirius red and Alcian blue are common methods for staining the extracellular matrix to identity fibroblasts and cartilage. Significant histological differences were observed between the modified and original groups at 6 weeks after implantation (Figure 8). The areas of hydrogel were filled with loose reticular red-stained fibroblast tissue or blue-stained cartilage tissue in the original groups. While the same areas in the modified groups were filled with regular dense fibrous or cartilage tissues, suggesting the PDA coating improved both chondrogenesis and fibrogenesis in vivo.

\section{DISCUSSION}

The goal of tissue engineering is to construct ideal scaffolds which can provide a microenvironment that facilitates the proliferation and differentiation of implanted cells by mimicking the natural $\mathrm{ECM}$ and that can provide suitable mechanical strength to meet the requirements of various tissues (Acri et al., 2019). The TMJ disc is a load-bearing tissue and subjected to complex mechanical forces during function, thus, the ability of an engineered disc to withstand anisotropic physiological forces is essential to successful regeneration. In this study, we fabricated a biomimetic composite system of 3D-printed synthetic polymers and AECM hydrogel for the reconstruction of TMJ disc. The compressive modulus of the PCL/PU and PU scaffolds was comparable to that of the central and peripheral zone of a native human TMJ disc, respectively. The PDA-coated composite constructs promoted chondrocyte and fibroblast attachment, proliferation, differentiation, and tissue regeneration. Therefore, the biomimetic composite system developed has the potential to be a promising substitute for damaged TMJ disc.

It has been reported that a tissue-specific ECM environment plays a critical role in regulating the cellular behaviors of cells, such as cell attachment, proliferation, and differentiation (Acri et al., 2019). In our prior study, porcine TMJ disc was processed into tissue-specific hydrogel that possessed outstanding biological properties and biocompatibility; however, weak mechanical strength prevented it from withstanding compression when implanted into a TMJ disc defect (Liang et al., 2020). Synthetic polymers are typically associated with improved mechanical properties and usually used as scaffolds to regenerate hard tissues (Aljohani et al., 2018). Recently, hybridization of natural and synthetic polymer materials is proven to be a promising approach to construct tissueengineered scaffolds which combines the advantages of both materials and meets diverse requirements (Setayeshmehr et al., 2019). This strategy has been applied to fabricate a variety of hybrid scaffolds to regenerate various hard tissues (Haaparanta et al., 2014; Chen et al., 2019a; Chen et al., 2019b). Therefore, in 

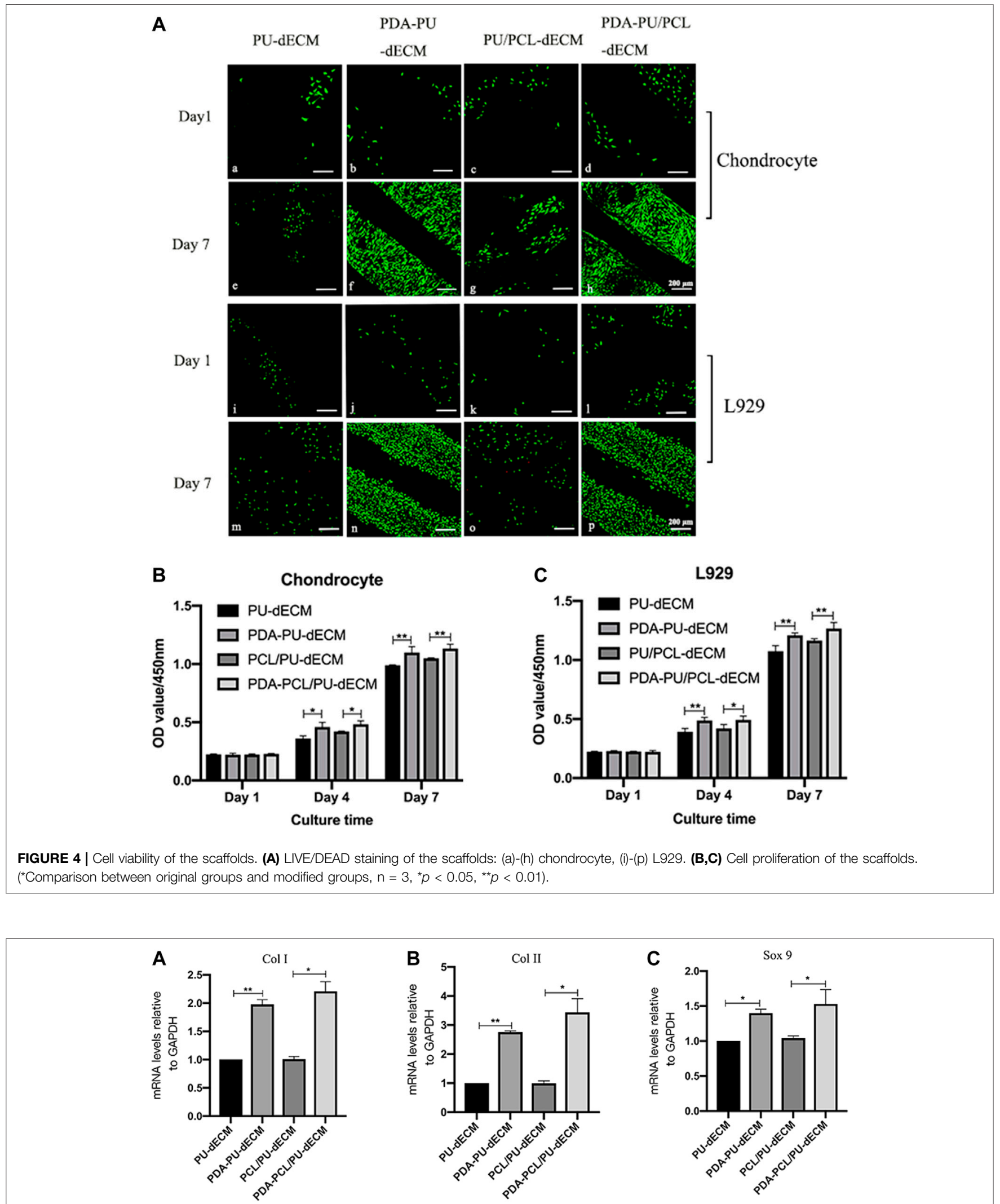

FIGURE 5| Genes expression of the scaffolds. (A) COL I, (B) COL II, and (C) $S O X 9$. $\left({ }^{\star}\right.$ Comparison between original groups and modified groups, $\mathrm{n}=3$, ${ }^{\star} p<0.05$, $\left.{ }^{* *} p<0.01\right)$ 

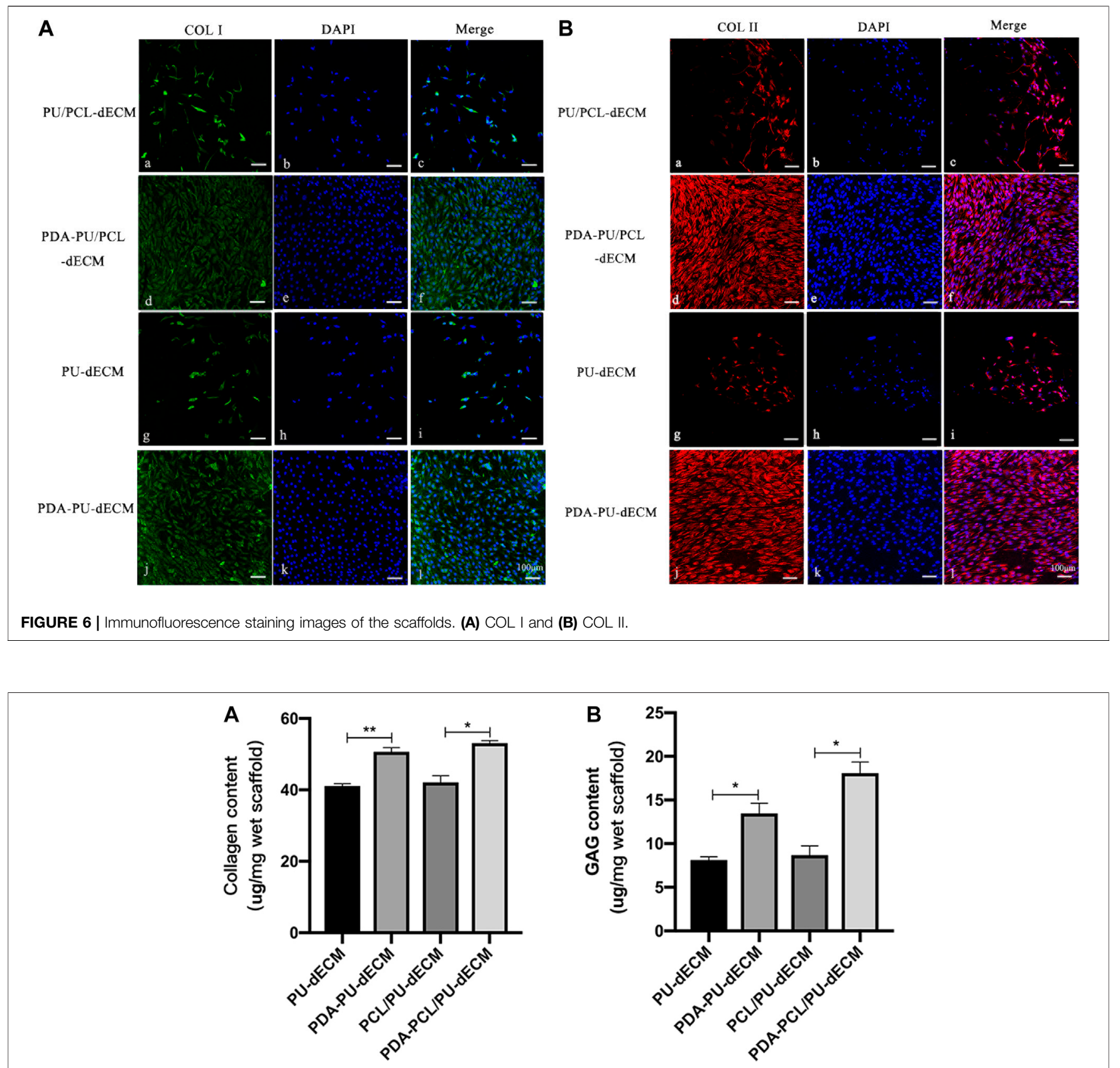

FIGURE 7 | Biochemical analysis for collagen and GAG quantification. (A) Collagen content of the scaffolds; (B) GAG content of the scaffolds. ( ${ }^{*}$ Comparison between original groups and modified groups, $n=3,{ }^{*} p<0.05,{ }^{* *} p<0.01$ ).

this study we chose polymer materials as the backbone of the composite scaffolds due to their excellent physical properties and TMJ disc-derived dECM hydrogel as a cell delivery system because of its superior biological properties, to engineer TMJ discs.

Compared with conventional approaches, 3D printing can produce specific implantable devices with architectural complexity that mimics that of the target tissue (Kumar et al., 2013). Although parameters vary according to the target tissue, pore sizes ranging from 200 to $350 \mu \mathrm{m}$ are necessary to promote cellular activities such as cell proliferation, migration, and differentiation (Kim et al., 2018; Li et al., 2019). Thus, we constructed 3D printed scaffolds with a pore size of about $350 \mu \mathrm{m}$. 3D printed PU and PCL scaffolds have been widely used in hard tissue engineering because of their excellent biological performance and desirable physical properties which can be tailored for specific applications (Wen et al., 2019; Han et al., 2020). However, PCL and PU exhibit poor cell attachment 

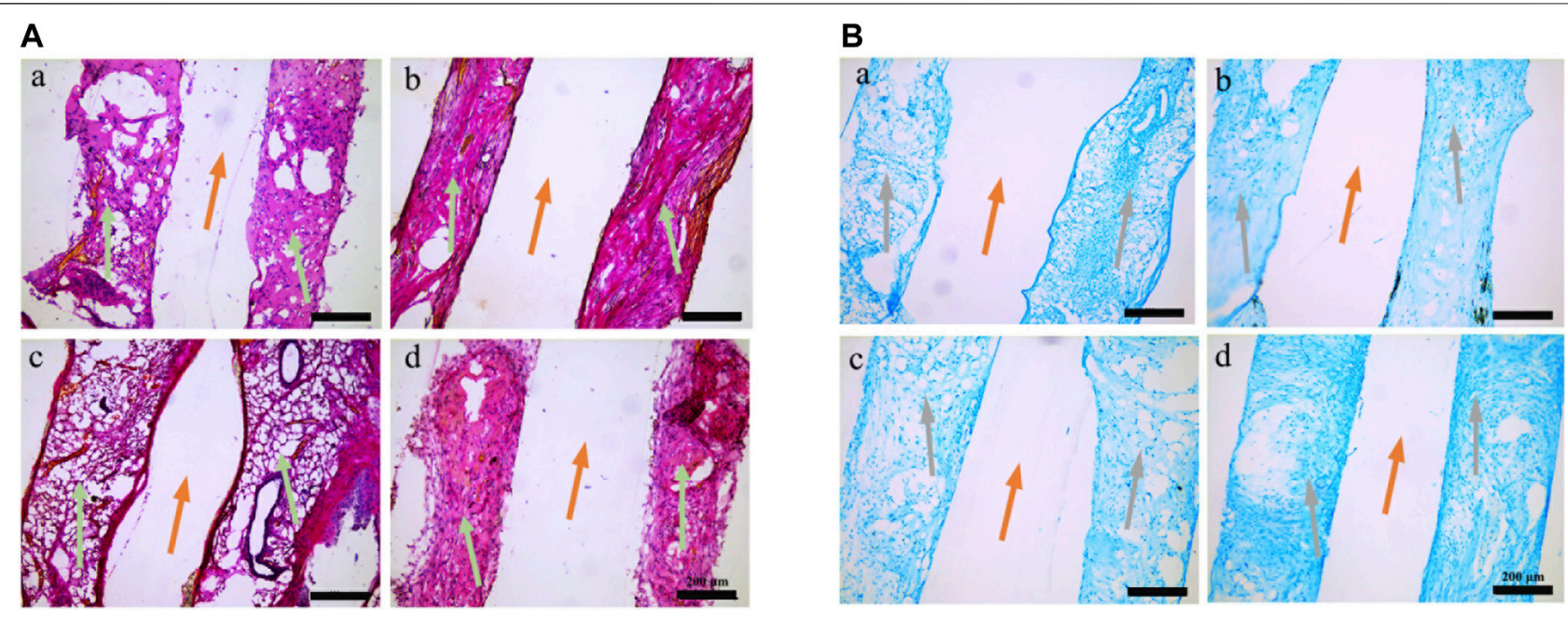

FIGURE 8| Histological analysis of the scaffolds retrieved from nude mice. (A) Picrosirius red staining. (B) Alcian blue staining. (a) PU-dECM; (b) PDA-PU- dECM; (c) PCL/PU-dECM; (d) PDA-PCL/PU- dECM. Orange arrows, 3D printed scaffolds framework lines; green arrows, collagen positively stained red with Picrosirius red; grey arrows, GAG positively stained blue with Alcian blue.

due to their hydrophobic properties and lack of biological cell recognition. Recently, it has been suggested that PDA coating can improve the hydrophobicity of PCL and PU and also significantly facilitate cell adhesion (Ghorbani et al., 2020). In this study, we modified the surface of the scaffolds with PDA coating to provide a more cell-friendly polymeric surface, and success of surface modification was confirmed by SEM and XPS. Previous studies indicated that increased surface roughness can be ascribed to the thorough distribution of submicron-sized particulates following PDA coating by atomic force microscopic analysis (Ju et al., 2011; Shin et al., 2011). The increased nitrogen/carbon ratio in modified groups was also confirmed by a previous study which revealed the presence of nitrogen peaks corresponding to primary amine groups present in the polydopamine structure (Shin et al., 2011). The decreased static contact angle in modified groups manifested that PDA coating led to improved hydrophilicity of the materials. Actually, the hydrophilicity can be increased independent of the underlying substrate's surface chemistry (Madhurakkat Perikamana et al., 2015). The enhanced mechanical properties in modified groups can be explained by the fact that DOPA contributes to cohesive strength through selfpolymerization, which improves the overall mechanical strength of the composite scaffold (Zhao et al., 2018). In addition, a $600 \mathrm{~nm}$ PDA layer formed on scaffold surface maintained the optimal pore size of scaffold to facilitate cell infiltration and nutrition supply (Ma et al., 2016). Therefore, PDA coating is a promising approach to functionalize biomaterials by changing their physiochemical properties.

Chondrocytes, fibroblasts, and a combination of both have been widely used to evaluate the suitability of scaffolds for fibrocartilage tissue repair and regeneration (Vanderploeg et al., 2004; Singh et al., 2011). Thus, in this study rat costal chondrocytes and L929 fibroblasts were used to evaluate the biofunctions and biocompatibility of the composite scaffolds in vitro and in vivo. The LIVE/DEAD test and CCK-8 assay showed that surface modification significantly enhanced cell adhesion and proliferation in modified groups. Previous studies have confirmed that the adhesion and proliferation of chondroblasts and fibroblasts can be improved by topological changes of the material surface (den Braber et al., 1995; Boyan et al., 1996). It was suggested that amine groups contained in PDA confer hydrophilicity and positive charge to substrates (Madhurakkat Perikamana et al., 2015). Furthermore, highly reactive amine groups can undergo secondary conjugation with various biomacromolecules in the culture medium and TMJ disc dECM hydrogel, which further facilitate cell adhesion and other biofunctions. Therefore, the deposition of polydopamine on the scaffold surface provided a favorable biomimetic ECM microenvironment, improving a range of cell metabolic functions.

The TMJ disc contains a heterogeneous collection of morphologically variable cells with characteristics of fibroblasts and chondrocytes. Selection of cell sources is of paramount importance for TMJ disc tissue engineering (Donahue et al., 2019). Besides various stem cells used to engineer the TMJ disc, the combination of costal cartilage and fibroblast was advocated to be a promising cell source due to relative tissue abundance and surgical accessibility (Huwe et al., 2018; Vapniarsky et al., 2018). In our study, the enhanced gene expression and protein deposition of collagens in modified groups were verified by $\mathrm{qPCR}$ and immunofluorescence staining. Collagen I and II are considered as the phenotypic markers of fibroblasts and chondrocytes, and the expression levels can be used to represent the differentiation and maturation of these two cells. As a pivotal transcription factor that plays a dominant role in chondrogenesis and chondrocyte differentiation pathways, Sox9 is required to enhance chondrocyte survival, maintain expression of cartilage-specific markers already active, such as Col II, and activate markers of overtly differentiated chondrocytes (Lefebvre and Dvir-Ginzberg, 
2017). The elevated expression of these tissue-specific markers in modified groups indicated the increased fibrogenesis and chondrogenesis following PDA coating. Collagen and GAG are the primary biochemical constituents that impart tensile and compressive strength to the TMJ disc (Lowe and Almarza, 2017). Thus, the higher level of collagen and GAG deposition can be associated to reinforced mechanical properties of the cell-laden composite scaffolds in the modified groups. Increased fibrogenesis and chondrogenesis in vivo can also be observed in the modified groups with Sirius red and Alcian blue staining. These histological results were consistent with our previous results which revealed enhanced cell adhesion, proliferation, differentiation, and maturation following PDA coating.

Although we have constructed a biomimetic cell-laden composite scaffold for engineering of TMJ disc, there are still some hurdles that need to be overcome to achieve ideal TMJ disc regeneration. First, polymeric scaffolds mimicking the complex geometric shape and region-specific mechanical properties of TMJ disc should be tailored by multiple-tool biofabrication strategies based on the image of MRI (Barbieri et al., 2020). Second, we have confirmed the fibrogenesis and chondrogenesis of the cell-laden composite scaffolds in nude mice; however, the successful regeneration of TMJ disc should be evaluated in physiologically mechanical environment by orthotopic implantation of these scaffolds in big animal model, such as mini pig (Helgeland et al., 2018). Third, the degradation velocity and mechanical strength of the polymeric scaffolds and hydrogel did not match each other perfectly, and these discrepancies probably compromised the biological and mechanical performance of the composite scaffolds in functional environment in vivo. Therefore, further study should focus on improving physical properties of these biomaterials to construct an ideal biomimetic substitute for TMJ disc tissue engineering (Kundu et al., 2015; Rothrauff et al., 2018).

\section{CONCLUSION}

In this study, a biomimetic composite scaffold was developed by combining 3D printed polymeric scaffolds and TMJ disc dECM hydrogel, and the biological and biomechanical properties of this scaffold were characterized. PDA-coated composite

\section{REFERENCES}

Abdala-Junior, R., Cortes, A. R. G., Aoki, E. M., Ferreira, S., Luz, J. G. C., Arita, E. S., et al. (2018). Impact of temporomandibular joint discectomy on condyle morphology: an animal study. J. Oral Maxillofac. Surg. 76 (5), 955 e951-955 e955. doi:10.1016/j.joms.2017.12.019

Acri, T. M., Shin, K., Seol, D., Laird, N. Z., Song, I., Geary, S. M., et al. (2019). Tissue engineering for the temporomandibular joint. Adv Healthc Mater. 8 (2), e1801236. doi:10.1002/adhm.201801236

Aljohani, W., Ullah, M. W., Zhang, X., and Yang, G. (2018). Bioprinting and its applications in tissue engineering and regenerative medicine. Int. J. Biol. Macromol. 107 (Part A), 261-275. doi:10.1016/j.ijbiomac.2017. 08.171 scaffolds possessed superior biomechanical properties and created a favorable microenvironment for cell adhesion, proliferation, differentiation, and maturation. The increased fibrogenesis and chondrogenesis of the cell-laden composite scaffolds in vitro and in vivo provided evidence of a promising strategy for TMJ disc regeneration. Overall, these results demonstrated that the biomimetic composite scaffold has therapeutic potential for TMJ disc engineering and regeneration.

\section{DATA AVAILABILITY STATEMENT}

The raw data supporting the conclusions of this article will be made available by the authors, without undue reservation.

\section{ETHICS STATEMENT}

The animal study was reviewed and approved by Guangzhou Medical University.

\section{AUTHOR CONTRIBUTIONS}

CL contributed to conception and design of the study; PY performed most of the experiments; JL carried out data analysis. All authors participated in drafting of the manuscript and critical revision of the draft. All authors have read and approved the final version of the manuscript.

\section{FUNDING}

This work was supported by the National Natural Science Foundation of China (31900946).

\section{ACKNOWLEDGMENTS}

The authors thank the Laboratory of Stomatology Hospital of Guangzhou Medical University for technical support.

Bahcecioglu, G., Hasirci, N., Bilgen, B., and Hasirci, V. (2019). A 3D printed PCL/ hydrogel construct with zone-specific biochemical composition mimicking that of the meniscus. Biofabrication 11 (2), 025002. doi:10.1088/1758-5090/aaf707

Barbero, A., Grogan, S., Schafer, D., Heberer, M., Mainil-Varlet, P., and Martin, I. (2004). Age related changes in human articular chondrocyte yield, proliferation and post-expansion chondrogenic capacity. Osteoarthritis Cartilage 12 (6), 476-484. doi:10.1016/j.joca.2004.02.010

Barbieri, A. A., Costa, A. L. F., Perez Gomes, J. P., Ricardo, A. L. F., Braz-Silva, P. H., and Lopes, S. (2020). Association of volume and voxel intensity of the articular disc and lateral pterygoid muscle in migraine patients: a study with magnetic resonance imaging. Acta Odontol. Scand. 78 (3), 189-195. doi:10.1080/ 00016357.2019.1676917

Bock, N., Pham, T. L., Nguyen, T. B., Nguyen, T. B., Tran, H. A., and Tran, P. A. (2020). Polydopamine coating of uncrosslinked chitosan as an acellular scaffold 
for full thickness skin grafts. Carbohydr. Polym. 245, 116524. doi:10.1016/j. carbpol.2020.116524

Bousnaki, M., Bakopoulou, A., Papadogianni, D., Barkoula, N. M., Alpantaki, K., Kritis, A., et al. (2018). Fibro/chondrogenic differentiation of dental stem cells into chitosan/alginate scaffolds towards temporomandibular joint disc regeneration. J. Mater. Sci. Mater. Med. 29 (7), 97. doi:10.1007/s10856-0186109-6

Boyan, B. D., Hummert, T. W., Dean, D. D., and Schwartz, Z. (1996). Role of material surfaces in regulating bone and cartilage cell response. Biomaterials. 17 (2), 137-146. doi:10.1016/0142-9612(96)85758-9

Brown, B. N., Chung, W. L., Almarza, A. J., Pavlick, M. D., Reppas, S. N., Ochs, M. W., et al. (2012). Inductive, scaffold-based, regenerative medicine approach to reconstruction of the temporomandibular joint disk. J. Oral Maxillofac. Surg. 70 (11), 2656-2668. doi:10.1016/j.joms.2011.12.030

Capellato, P., Camargo, S. E. A., and Sachs, D. (2020). Biological response to nanosurface modification on metallic biomaterials. Curr. Osteoporos. Rep. 18 (6), 790-795. doi:10.1007/s11914-020-00635-x

Chen, M., Feng, Z., Guo, W., Yang, D., Gao, S., Li, Y., et al. (2019a). PCL-MECMbased hydrogel hybrid scaffolds and meniscal fibrochondrocytes promote whole meniscus regeneration in a rabbit meniscectomy model. ACS Appl. Mater. Interfaces 11 (44), 41626-41639. doi:10.1021/acsami.9b13611

Chen, Y., Lee, K., Kawazoe, N., Yang, Y., and Chen, G. (2019b). PLGA-collagenECM hybrid scaffolds functionalized with biomimetic extracellular matrices secreted by mesenchymal stem cells during stepwise osteogenesis-coadipogenesis. J. Mater. Chem. B. 7 (45), 7195-7206. doi:10.1039/c9tb01959f

Daly, A. C., Freeman, F. E., Gonzalez-Fernandez, T., Critchley, S. E., Nulty, J., and Kelly, D. J. (2017). 3D bioprinting for cartilage and osteochondral tissue engineering. Adv Healthc Mater. 6 (22), 1700298. doi:10.1002/adhm.201700298

den Braber, E. T., de Ruijter, J. E., Smits, H. T., Ginsel, L. A., von Recum, A. F., and Jansen, J. A. (1995). Effect of parallel surface microgrooves and surface energy on cell growth. J. Biomed. Mater. Res. 29 (4), 511-518. doi:10.1002/jbm. 820290411

Dimitroulis, G. (2011). A critical review of interpositional grafts following temporomandibular joint discectomy with an overview of the dermis-fat graft. Int. J. Oral Maxillofac. Surg. 40 (6), 561-568. doi:10.1016/j.ijom.2010. 11.020

Dolwick, M. F. (2007). Temporomandibular joint surgery for internal derangement. Dent. Clin. 51 (1), 195-208. doi:10.1016/j.cden.2006.10.003

Donahue, R. P., Hu, J. C., and Athanasiou, K. A. (2019). Remaining hurdles for tissue-engineering the temporomandibular joint disc. Trends Mol. Med. 25 (3), 241-256. doi:10.1016/j.molmed.2018.12.007

Eiji, T., and Theo, V. E. (2003). Biomechanical behavior of the temporomandibular joint disc. Crit. Rev. Oral Biol. Med. 14 (2), 138-150.

Eriksson, L., and Westesson, P. L. (1992). Temporomandibular joint diskectomy. No positive effect of temporary silicone implant in a 5-year follow-up. Oral Surg. Oral Med. Oral Pathol. 74 (3), 259-272. doi:10.1016/0030-4220(92) 90056-v

Ghorbani, F., Ghalandari, B., Khan, A. L., Li, D., Zamanian, A., and Yu, B. (2020). Decoration of electrical conductive polyurethane-polyaniline/polyvinyl alcohol matrixes with mussel-inspired polydopamine for bone tissue engineering. Biotechnol. Prog. 36 (6), e3043. doi:10.1002/btpr.3043

Godoy-Gallardo, M., Portoles-Gil, N., Lopez-Periago, A. M., Domingo, C., and Hosta-Rigau, L. (2020). Multi-layered polydopamine coatings for the immobilization of growth factors onto highly-interconnected and bimodal PCL/HA-based scaffolds. Mater Sci. Eng. C Mater Biol. Appl. 117, 111245. doi:10.1016/j.msec.2020.111245

Gonçalves, D., Camparis, C., Speciali, J., Franco, A., Castanharo, S., and Bigal, M. (2011). Temporomandibular disorders are differentially associated with headache diagnoses: a controlled study. Clin. J. Pain 27 (7), 611-615. doi:10. 1097/AJP.0b013e31820e12f5

Haaparanta, A. M., Jarvinen, E., Cengiz, I. F., Ella, V., Kokkonen, H. T., Kiviranta, I., et al. (2014). Preparation and characterization of collagen/PLA, chitosan/ PLA, and collagen/chitosan/PLA hybrid scaffolds for cartilage tissue engineering. J. Mater. Sci. Mater. Med. 25 (4), 1129-1136. doi:10.1007/ s10856-013-5129-5

Han, Y., Lian, M., Sun, B., Jia, B., Wu, Q., Qiao, Z., et al. (2020). Preparation of high precision multilayer scaffolds based on Melt Electro-Writing to repair cartilage injury. Theranostics 10 (22), 10214-10230. doi:10.7150/thno.47909
Helgeland, E., Shanbhag, S., Pedersen, T. O., Mustafa, K., and Rosen, A. (2018). Scaffold-based temporomandibular joint tissue regeneration in experimental animal models: a systematic review. Tissue Eng. B Rev. 24 (4), 300-316. doi:10. 1089/ten.TEB.2017.0429

Huwe, L. W., Brown, W. E., Hu, J. C., and Athanasiou, K. A. (2018). Characterization of costal cartilage and its suitability as a cell source for articular cartilage tissue engineering. J Tissue Eng Regen Med. 12 (5), 1163-1176. doi:10.1002/term.2630

Jo, S., Kang, S. M., Park, S. A., Kim, W. D., Kwak, J., and Lee, H. (2013). Enhanced adhesion of preosteoblasts inside 3D PCL scaffolds by polydopamine coating and mineralization. Macromol. Biosci. 13 (10), 1389-1395. doi:10.1002/mabi. 201300203

Ju, K. Y., Lee, Y., Lee, S., Park, S. B., and Lee, J. K. (2011). Bioinspired polymerization of dopamine to generate melanin-like nanoparticles having an excellent free-radical-scavenging property. Biomacromolecules 12 (3), 625-632. doi:10.1021/bm101281b

Kim, J. Y., Ahn, G., Kim, C., Lee, J. S., Lee, I. G., An, S. H., et al. (2018). Synergistic effects of beta tri-calcium phosphate and porcine-derived decellularized bone extracellular matrix in 3D-printed polycaprolactone scaffold on bone regeneration. Macromol. Biosci. 18 (6), e1800025. doi:10.1002/mabi. 201800025

Kobayashi, E., Nakahara, T., Inoue, M., Shigeno, K., and Nakamura, T. (2015). Experimental study on in situ tissue engineering of the temporomandibular joint disc using autologous bone marrow and collagen sponge scaffold. J. Hard Tissue Biol. 24 (2), 211-218. doi:10.2485/jhtb.24.211

Kumar, A., Webster, T. J., Biswas, K., and Basu, B. (2013). Flow cytometry analysis of human fetal osteoblast fate processes on spark plasma sintered hydroxyapatite-titanium biocomposites. J. Biomed. Mater. Res. 101 (10), 2925-2938. doi:10.1002/jbm.a.34603

Kundu, J., Shim, J. H., Jang, J., Kim, S. W., and Cho, D. W. (2015). An additive manufacturing-based PCL-alginate-chondrocyte bioprinted scaffold for cartilage tissue engineering. J Tissue Eng Regen Med. 9 (11), 1286-1297. doi: $10.1002 /$ term. 1682

Lee, C. H., Rodeo, S. A., Fortier, L. A., Lu, C., Erisken, C., and Mao, J. J. (2014). Protein-releasing polymeric scaffolds induce fibrochondrocytic differentiation of endogenous cells for knee meniscus regeneration in sheep. Sci. Transl. Med. 6 (266), 266ra171. doi:10.1126/scitranslmed.3009696

Lefebvre, V., and Dvir-Ginzberg, M. (2017). SOX9 and the many facets of its regulation in the chondrocyte lineage. Connect. Tissue Res. 58 (1), 2-14. doi:10. 1080/03008207.2016.1183667

Legemate, K., Tarafder, S., Jun, Y., and Lee, C. (2016). Engineering human TMJ discs with protein-releasing 3D-printed scaffolds. J. Dent. Res. 95 (7), 800-807. doi:10.1177/0022034516642404

Li, J., Yao, Q., Xu, Y., Zhang, H., Li, L. L., and Wang, L. (2019). Lithium chloridereleasing 3D printed scaffold for enhanced cartilage regeneration. Med. Sci. Monit. 25, 4041-4050. doi:10.12659/MSM.916918

Liang, J., Yi, P., Wang, X., Huang, F., Luan, X., Zhao, Z., et al. (2020). Acellular matrix hydrogel for repair of the temporomandibular joint disc. J. Biomed. Mater. Res. B Appl. Biomater. 108 (7), 2995-3007. doi:10.1002/jbm.b.34629

Lim, P. F., and Sbhalang, S. (2010). Development of temporomandibular disorders is associated with greater bodily pain experience. Clin. J. Pain 26 (2), 116-120. doi:10.1097/AJP.0b013e3181c507ef

Lowe, J., and Almarza, A. J. (2017). A review of in-vitro fibrocartilage tissue engineered therapies with a focus on the temporomandibular joint. Arch. Oral Biol. 83, 193-201. doi:10.1016/j.archoralbio.2017.07.013

Ma, H., Luo, J., Sun, Z., Xia, L., Shi, M., Liu, M., et al. (2016). 3D printing of biomaterials with mussel-inspired nanostructures for tumor therapy and tissue regeneration. Biomaterials 111, 138-148. doi:10.1016/j.biomaterials.2016. 10.005

Madhurakkat Perikamana, S. K., Lee, J., Lee, Y. B., Shin, Y. M., Lee, E. J., Mikos, A. G., et al. (2015). Materials from mussel-inspired chemistry for cell and tissue engineering applications. Biomacromolecules 16 (9), 2541-2555. doi:10.1021/ acs.biomac.5b00852

Miloro, M., and Henriksen, B. (2010). Discectomy as the primary surgical option for internal derangement of the temporomandibular joint. J. Oral Maxillofac. Surg. 68 (4), 782-789. doi:10.1016/j.joms.2009.09.091

Moura, C., Trindade, D., Vieira, M., Francisco, L., Angelo, D. F., and Alves, N. (2020). Multi-material implants for temporomandibular joint disc repair: 
tailored additive manufacturing production. Front Bioeng Biotechnol. 8, 342. doi:10.3389/fbioe.2020.00342

Pina, S., Ribeiro, V. P., Marques, C. F., Maia, F. R., Silva, T. H., Reis, R. L., et al. (2019). Scaffolding strategies for tissue engineering and regenerative medicine applications. Materials 12 (11). doi:10.3390/ma12111824

Qian, Y., Zhao, X., Han, Q., Chen, W., Li, H., and Yuan, W. (2018). An integrated multi-layer 3D-fabrication of PDA/RGD coated graphene loaded PCL nanoscaffold for peripheral nerve restoration. Nat. Commun. 9 (1), 323. doi:10.1038/s41467-017-02598-7

Qiu, W. Z., Yang, H. C., and Xu, Z. K. (2018). Dopamine-assisted co-deposition: an emerging and promising strategy for surface modification. Adv. Colloid Interface Sci. 256, 111-125. doi:10.1016/j.cis.2018.04.011

Renapurkar, S. K. (2018). Discectomy versus disc preservation for internal derangement of the temporomandibular joint. Oral Maxillofac. Surg. Clin. 30 (3), 329-333. doi:10.1016/j.coms.2018.05.002

Richbourg, N. R., Peppas, N. A., and Sikavitsas, V. I. (2019). Tuning the biomimetic behavior of scaffolds for regenerative medicine through surface modifications. J. Tissue Eng Regen Med. 13 (8), 1275-1293. doi:10.1002/term.2859

Romanazzo, S., Vedicherla, S., Moran, C., and Kelly, D. J. (2018). Meniscus ECMfunctionalised hydrogels containing infrapatellar fat pad-derived stem cells for bioprinting of regionally defined meniscal tissue. J. Tissue Eng Regen Med. 12 (3), e1826-e1835. doi:10.1002/term.2602

Rothrauff, B. B., Coluccino, L., Gottardi, R., Ceseracciu, L., Scaglione, S., Goldoni, L., et al. (2018). Efficacy of thermoresponsive, photocrosslinkable hydrogels derived from decellularized tendon and cartilage extracellular matrix for cartilage tissue engineering. J. Tissue Eng. Regen. Med. 12 (1), e159-e170. doi:10.1002/term.2465

Setayeshmehr, M., Esfandiari, E., Rafieinia, M., Hashemibeni, B., Taheri-Kafrani, A., Samadikuchaksaraei, A., et al. (2019). Hybrid and composite scaffolds based on extracellular matrices for cartilage tissue engineering. Tissue Eng. B Rev. 25 (3), 202-224. doi:10.1089/ten.TEB.2018.0245

Shin, Y. M., Lee, Y. B., and Shin, H. (2011). Time-dependent mussel-inspired functionalization of poly(L-lactide-co-varepsilon-caprolactone) substrates for tunable cell behaviors. Colloids Surf. B Biointerfaces 87 (1), 79-87. doi:10.1016/j. colsurfb.2011.05.004

Singh, M., Pierpoint, M., Mikos, A. G., and Kasper, F. K. (2011). Chondrogenic differentiation of neonatal human dermal fibroblasts encapsulated in alginate beads with hydrostatic compression under hypoxic conditions in the presence of bone morphogenetic protein-2. J. Biomed. Mater. Res. 98 (3), 412-424. doi:10.1002/jbm.a.33129

Tamay, D. G., Dursun Usal, T., Alagoz, A. S., Yucel, D., Hasirci, N., and Hasirci, V. (2019). 3D and 4D printing of polymers for tissue engineering applications. Front. Bioeng. Biotechnol. 7, 164. doi:10.3389/fbioe.2019.00164

Tarafder, S., Koch, A., Jun, Y., Chou, C., Awadallah, M. R., and Lee, C. H. (2016). Micro-precise spatiotemporal delivery system embedded in 3D printing for complex tissue regeneration. Biofabrication 8 (2), 025003. doi:10.1088/17585090/8/2/025003
Theodoridis, K., Aggelidou, E., Manthou, M., Demiri, E., Bakopoulou, A., and Kritis, A. (2019). Assessment of cartilage regeneration on 3D collagenpolycaprolactone scaffolds: evaluation of growth media in static and in perfusion bioreactor dynamic culture. Colloids Surf. B Biointerfaces 183, 110403. doi:10.1016/j.colsurfb.2019.110403

Vanderploeg, E. J., Imler, S. M., Brodkin, K. R., Garcia, A. J., and Levenston, M. E. (2004). Oscillatory tension differentially modulates matrix metabolism and cytoskeletal organization in chondrocytes and fibrochondrocytes. J. Biomech. 37 (12), 1941-1952. doi:10.1016/j.jbiomech.2004.02.048

Vapniarsky, N., Huwe, L. W., Arzi, B., Houghton, M. K., Wong, M. E., Wilson, J. W., et al. (2018). Tissue engineering toward temporomandibular joint disc regeneration. Sci. Transl. Med. 10 (446). doi:10.1126/scitranslmed.aaq1802

Wang, C., Wang, S., Zhang, B., Zhang, X., Tong, X., Peng, H., et al. (2018). Layering Poly (lactic-co-glycolic acid)-based electrospun membranes and co-culture cell sheets for engineering temporomandibular joint disc. J. Biol. Regul. Homeost. Agents. 32 (1), 55-61.

Wei, P., Xu, Y., Gu, Y., Yao, Q., Li, J., and Wang, L. (2020). IGF-1-releasing PLGA nanoparticles modified 3D printed PCL scaffolds for cartilage tissue engineering. Drug Deliv. 27 (1), 1106-1114. doi:10.1080/10717544.2020. 1797239

Wen, Y. T., Dai, N. T., and Hsu, S. H. (2019). Biodegradable water-based polyurethane scaffolds with a sequential release function for cell-free cartilage tissue engineering. Acta. Biomater. 88, 301-313. doi:10.1016/j. actbio.2019.02.044

Wu, Y., Gong, Z., Li, J., Meng, Q., Fang, W., and Long, X. (2014). The pilot study of fibrin with temporomandibular joint derived synovial stem cells in repairing TMJ disc perforation. BioMed Res. Int. 2014, 454021. doi:10.1155/2014/454021

Zhao, X. G., Hwang, K.-J., Lee, D., Kim, T., and Kim, N. (2018). Enhanced mechanical properties of self-polymerized polydopamine-coated recycled PLA filament used in 3D printing. Appl. Surf. Sci. 441, 381-387. doi:10.1016/j.apsusc. 2018.01.257

Zhu, Y., Gao, C., and Shen, J. (2002). Surface modification of polycaprolactone with poly(methacrylic acid) and gelatin covalent immobilization for promoting its cytocompatibility. Biomaterials 23 (24), 4889-4895. doi:10.1016/s01429612(02)00247-8

Conflict of Interest: The authors declare that the research was conducted in the absence of any commercial or financial relationships that could be construed as a potential conflict of interest.

Copyright $\odot 2021$ Yi, Liang, Huang, Zhao, Zhou, Gao, Huang, Chen, Guo and Liu. This is an open-access article distributed under the terms of the Creative Commons Attribution License (CC BY). The use, distribution or reproduction in other forums is permitted, provided the original author(s) and the copyright owner(s) are credited and that the original publication in this journal is cited, in accordance with accepted academic practice. No use, distribution or reproduction is permitted which does not comply with these terms. 